\title{
Análisis ambiental desde una perspectiva jurídica del ecosistema sur del Municipio La Sierpe
}

\author{
Alexis Ramón Zedeño Valmaseda ${ }^{1}$ \\ Bertha Emérita Carbonell Nuñez ${ }^{2}$ \\ Roberto Luis Elizalde Consuegra ${ }^{3}$
}

\section{Resumen}

El trabajo es un resultado de investigaciones realizadas en el marco del proyecto Formación de capacidades para el manejo integrado de la costa Sur del municipio La Sierpe. En el mismo se hace un análisis ambiental a partir de un marco jurídico nacional e internacional vigente de las afectaciones provocadas por la no existencia de un Manejo integrado de la costa sur del municipio la Sierpe. El análisis se centra principalmente en tres recursos fundamentales que son los que más se ven afectados, los cuales son: Los bosques, las aguas terrestres y marinas y los suelos como uno de los componentes fundamentales de la tierra. En el trabajo se analizan detalladamente las causas y condiciones que han propiciado el deterioro de estos recursos, así como los elementos legales que la sustentan. Todo lo cual propicia un importante material investigativo a tener en cuenta por las autoridades del territorio para la toma de decisiones en cuanto a la necesidad de implementar el manejo integrado de la zona. Desde el punto de vista investigativo se realiza un diagnóstico sobre las principales afectaciones ambientales de la zona, así como las acusas que la originan a partir de lo cual la ciencia puede actuar con propuestas para mitigar estos cambios.

Palabras clave: Manejo integrado; zona costera; bosques; deforestación; marco jurídico; daño ambiental.

\section{Summary}

The work is a result of investigations carried out in the mark of the project Formation of capacities for the integrated handling of the south coast of the municipality The Serpent. In the same one an environmental analysis is made starting from an effective national and international juridical mark of the affectations caused by the non existence of an integrated Handling of the south coast of the municipality the Serpent. The analysis is centered mainly in three fundamental resources that are those that more they are affected, which are: The forests, the terrestrial and marine waters, the floors like one of the fundamental components of the earth. In the work they

\footnotetext{
Ms. C. Director de Centro Universitario. Universidad José Martí de Sancti Spiritus. alexisz@uniss.edu.cu

Lic. Profesora. Universidad José Martí de Sancti Spíritus. berthica@uniss.edu.cu

Ms. C. Profesor. Universidad José Martí de Sancti Spiritus. relizalde@fach.uniss.edu.cu
} 
are analyzed the causes and conditions that have propitiated the deterioration of these resources detailedly as well as the legal elements that sustain it. All that which favorable an important investigative material to keep in mind for the authorities of the territory for the taking of decisions as for the necessity of implementing the integrated handling of the area. From the investigative point of view he/she is carried out a diagnosis on the main environmental affectations of the area as well as you accuse them that they originate it starting from that which the science can act with proposals to mitigate these changes.

Keywords: I manage integrated; coastal area; forests; desforestación; juridical mark; I damage environmental.

\section{Introducción}

La Sierpe constituye el municipio más joven de Cuba, surge en 1976 como consecuencia de la nueva división político-administrativa. Posee límites definidos, tanto por accidentes geográficos como por elementos estructurales creados por el hombre. Limita al oeste con el municipio de Sancti Spiritus, por el norte con Jatibonico, por el este con los municipios de Majagua y Venezuela de la provincia Ciego de Ávila, y por el sur con el mar Caribe. Su extensión territorial es de $1035 \mathrm{~km}^{2}$, representando dentro de la provincia el 15,3\% de sus áreas, lo que lo sitúa como el tercer municipio más grande de la provincia, sólo superado por Sancti Spiritus y Trinidad.

El municipio tiene $56 \mathrm{~km}$ de costas, caracterizados por predominar en ellos zonas cenagosas originadas por la poca profundidad de su plataforma marina. Sus playas son escasas, y no se les da ningún tipo de aprovechamiento recreativo ni económico. Estas costas pertenecen al tramo María Aguilar-Cabo Santa Cruz y sus formas son regulares.

Entre sus accidentes costeros se destacan Punta Colorada, Punta Manatí, Punta de Media, Punta de Paso Banao, Punta Tunas, Ensenada de Las Guásimas, Ensenada de Juan Hernández, Ensenada de Las Canarias y delta del río Zaza. No existe en su configuración bahías, penínsulas, golfos y cabos, y por ser una costa baja y pantanosa se localizan muy cerca del litoral, manifestaciones cálcicas formadas durante la era cenozoica.

La actividad económica del municipio La Sierpe se basa en la agricultura, dedicado en lo fundamental a la cosecha de arroz para el consumo del balance nacional, asociado a las producciones de cultivos varios como hortalizas, algunos frutales y pequeñas granjas productoras de viandas para el autoconsumo del municipio, además de la ganadería dedicada en la actualidad al desarrollo del ganado lechero para satisfacer las demandas del municipio e incrementar el acopio al combinado lácteo de la provincia, por lo que se puede considerar un municipio mono productor; sin embargo, cuando 
nos ubicamos en el contexto del territorio podemos apreciar que existen $56 \mathrm{~km}$ de costa que se encuentran sub-utilizados y pudieran contribuir al desarrollo de otros importantes renglones productivos y sociales. La falta de capacitación sobre el manejo de los sistemas costeros en el territorio ha impedido que el mismo sea aprovechado eficientemente en función del desarrollo endógeno.

El municipio no cuenta con áreas recreativas de baños, dígase playas, campismos, etc. donde la población pueda acudir a saciar sus necesidades recreativas y cada verano el Complejo Agroindustrial Arrocero (CAI) invierte numerosos recursos para trasladar y atender a sus más de 3000 trabajadores en una Villa que se encuentra a más de $100 \mathrm{~km}$ de distancia y fuera de los límites del municipio, así también el Consejo de la Administración municipal tiene que acudir al apoyo de otros municipios para garantizar capacidades en unidades del campismo popular por no contar con propias del territorio.

La explotación de los potenciales que aporta nuestra cuenca marina es asumida por otros territorios de la provincia, (Trinidad y Tunas de Zaza) por no contar el territorio con una infraestructura capaz de asumir la captura y comercialización de los productos del mar. Cada año kilómetros de tierra adentro son afectados por la salinización por no existir una estrategia para un manejo adecuado de la misma, lo cual ha ocasionado la perdida de la productividad en un número importante de caballerías que antiguamente eran destinadas al cultivo del arroz.

El manejo medioambiental de la franja se ha visto afectado por la falta de acciones encaminadas a este fin lo que ha traído consigo la extinción de importantes especies animales y vegetales de la zona con las consiguientes afectaciones al medio ambiente. El municipio cuenta con 6 Consejos populares de los cuales 2 (Las Nuevas y Mapos) son considerados los más vulnerables, ya que los mismos son los que se encuentran más alejados de la cabecera municipal siendo difícil el acceso a los mismos y por otro lado por las condiciones socio-culturales desfavorable que presentan los mismos, además coincidentemente son los dos Consejos que se encuentran más cerca de nuestra franja costera.

Esta investigación constituye una tarea del proyecto "Formación de capacidades para el manejo integrado del ecosistema costero y sus comunidades en el municipio de La Sierpe". El cual es coordinado por el Centro Universitario municipal de la Sierpe, perteneciente a la Universidad de Sancti Spiritus “José Martí Pérez". 


\section{Revisión de literatura}

\section{Marco jurídico para el manejo integrado de las zonas costeras a nivel internacional}

El Derecho Ambiental Internacional reconoce dentro de sus principios fundamentales la regulación jurídica integral. Con este principio se logra la armonización y unificación de las legislaciones a nivel internacional, con los regímenes jurídicos de los diferentes Estados y regiones de la Tierra en relación a las normas jurídicas ambientales internacionales destinadas a la prevención, represión, defensa, conservación, mejoramiento y restauración. Incluye la perspectiva macroscópica e integradora del ambiente, debido a la fragmentación de las normas ambientales.

(Pigretti, 1992) plantea que algunos Estados antes de la Conferencia de Estocolmo efectuada en el año 1972, respondían a criterios no sistemáticos ni epistemológicos alguno, sino a una necesidad de legislar dentro de las ramas clásicas del derecho determinadas conductas que requerían legislación especial.

Como elemento referencial de este principio se advierte que a finales de la década de los sesenta, hasta los primeros años de la década de los setenta, se produjo una alarma lanzada por grupos científicos, aportando argumentos en relación con la problemática medioambiental, que alcanzó gran fuerza en algunos países, entre los que se destacaron Estados Unidos, Francia y Alemania. Paralelo a ello la Asamblea General de la Naciones Unidas, mediante la Resolución 2398 de fecha 3 de Diciembre de 1968, convocó a la celebración de la Conferencia de las Naciones Unidas sobre el Medio Ambiente Humano, llamada Conferencia de Estocolmo, en la cual se enfatizó en los temas ambientales, fundamentalmente en los asuntos oceánicos y costeros, lo que constituyó una expresión del mencionado principio, válido en los análisis relacionados con el tratamiento jurídico internacional.

En la Segunda Conferencia de las Naciones Unidas, llamada también Cumbre de Río o Cumbre de la Tierra, se establecieron como premisas esenciales: la observación y evaluación profunda de toda una serie de problemas mundiales existentes en los años 8o. A partir de esta Cumbre toma fuerzas el concepto de "manejo integrado de las zonas costeras", al recoger dentro de los principales programas de áreas el manejo integrado y el desarrollo sostenible de las zonas costeras. La celebración de esta Conferencia impulsó a los países que firmaron este documento a dirigir sus acciones hacia este nuevo enfoque integrador, definiendo las principales metas y acciones del mismo.

Luego en el año 2002 se celebró la Cumbre sobre Desarrollo Sostenible, al haber transcurrido diez años de la Declaración de Río sobre la Tierra, para la evaluación de los avances sobre el Medio Ambiente, su desarrollo y sostenibilidad. Su documento rector es la Declaración de Johannesburgo del 2002, en la cual quedó confirmado el 
diseño, aprobación e implementación, de programas y planes de manejo, sustentados en normas jurídicas ajustadas a la pirámide normativa de cada Estado.

\section{Tratamiento legal del manejo integrado de las zonas costeras en Cuba}

Con el triunfo de Revolución en Cuba, 1959, y en correspondencia con las transformaciones del régimen de propiedad privada hacia la propiedad social, se advierte dentro de las principales medidas económicas y sociales que se adoptan, la voluntad estatal en la preservación del medio ambiente y sus ecosistemas incluyendo el tratamiento de las zonas costeras. En el ámbito jurídico la promulgación de la Constitución de la República en 1976, reconoció la protección del medioambiente como un derecho y un deber nacional.

En 1992, se modifica el Artículo 27 de la mencionada Constitución, quedando textualmente redactado:

El Estado protege al medio ambiente y los recursos naturales del país. Reconoce su estrecha vinculación con el desarrollo económico y social sostenible para hacer más racional la vida humana y asegurar la supervivencia, el bienestar y la seguridad de las generaciones actuales y futuras. Corresponde a los órganos competentes aplicar ésta política. Es deber de los ciudadanos contribuir a la protección del agua, la atmósfera, la conservación del suelo, la flora, la fauna y de todo el uso potencial de la naturaleza.

Desde el punto de vista del proceso de institucionalización derivado de los preceptos constitucionales, en 1976 se promulgó la Ley de Organización de la Administración Central del Estado, que confirió al Comité Estatal de Ciencia y Técnica el establecimiento, la dirección y el control del Sistema Nacional de Protección del Medio Ambiente y el Uso Racional de los Recursos Naturales. Por acuerdo del órgano ejecutivo del gobierno de esta misma fecha, se creó la Comisión Nacional de Protección del Medio Ambiente y Conservación de los Recursos Naturales, lo que constituyó el primer antecedente en materia de institucionalización en materia medioambiental en Cuba.

En 1980 mediante el Decreto Ley No.31 de 10 de enero de ese año la Academia de Ciencias de Cuba recibió las funciones atribuidas al Comité Estatal de Ciencia y Técnica, adscribiéndose a ella igualmente la comisión Nacional de Protección del Medio Ambiente y Conservación de los Recursos Naturales. El 10 de enero de 1981, al promulgarse la Ley No 33 "De Protección del Medio Ambiente y el Uso Racional de los Recursos Naturales", se dispuso la creación de un Sistema Nacional de Protección del Medio Ambiente y del Uso Racional de los Recursos Naturales y de una Comisión Nacional que sería la instancia coordinadora de la gestión ambiental. 
En Cuba a partir de 1992, en correspondencia con los acuerdos de la Cumbre de Río, se produjeron cambios institucionales dirigidos al establecimiento e implementación con mayor eficacia de la política ambiental. Se sustituyó a la Academia de Ciencias del Sistema de Organismos de la Administración Central del Estado, al promulgarse el Decreto Ley No.147/ 1994 "De la reorganización de la Administración Central del Estado", con ello se extinguía la Comisión Nacional de Protección del Medio Ambiente y Uso Racional de los Recursos Naturales, cuyas funciones fueron transferidas al Ministerio de Ciencia Tecnología y Medio Ambiente, en lo adelante, CITMA, que entre otras serían, dirigir, ejecutar y controlar la política del Estado y el Gobierno en materia ambiental.

A pesar de las normativas jurídicas puestas en vigor en la etapa revolucionaria, así como la constitución y funcionamiento de instituciones para atender el medio ambiente, no se advirtieron cambios sustanciales en el cumplimiento de la política medioambiental. El tratamiento de los temas ambientales incluyendo las zonas costeras, se ejecutaba y controlaba por cada uno de los sectores con sus propias disposiciones, adoleciendo de un instrumento jurídico que de manera diferenciada definiera los principios y el alcance para la gestión y el tratamiento integrado de las zonas costeras.

\section{Manejo integrado de las zonas costeras en correspondencia con el Decreto Ley 212/2000 "Gestión de las zonas costeras"}

En correspondencia con los principios de supletoriedad y de especialidad de las normas para dar debida coherencia al sistema jurídico con el contenido especializado con relación a leyes de contenido general y la regulación de aspectos específicos en la política ambiental, se pone en vigor el Decreto Ley 212/200o "Gestión de las zonas costeras". Dispone la delimitación de la extensión de la zona costera a partir de las características fisiográficas de cada tipo de costa. Añade las acciones que garantizan la protección y el uso sostenible de la zona costera y su zona de protección, en atención a los principios del manejo integrado de la zona costera.

El Decreto-Ley 212/ 2000, permitió un vuelco a las actividades y acciones que sobre la zona costera se venían realizando en Cuba, puesto que incluye el desarrollo sostenible en las actividades en la zona costera. Incorpora los principios del manejo integrado costero en los planes de ordenamiento territorial y urbanístico, y en los esquemas de desarrollo del turismo y la integración del conocimiento científico adquirido al estudio de las condiciones fisiográficas de la zona costera. La interpretación del contenido de las regulaciones de esta norma jurídica permite comprender que el manejo integrado de las zonas costeras, no se limita a la extensión territorial reducida a la que se refieren estos preceptos al definir la zona costera.

Prevé que de acuerdo a la identificación y evaluación que se haya realizado de las problemáticas ambientales que presentan los ecosistemas, se puede establecer como 
asuntos claves un programa de manejo, elementos del contexto social, ambiental e institucional que no necesariamente estén constreñidos a una franja tan precisa y estrecha de interacción entre el agua, la tierra, la atmósfera y el mar. El manejo integrado de las zonas costeras tiene que actuar sobre todas las causas que generan los problemas en ese lugar, con independencia del lugar en que estos se generen. Determina que el elemento político institucional, no puede limitarse al área geográfica de las costas.

\section{Materiales y métodos}

El trabajo fue realizado tomando como referencia los 56 kilómetros de costa con que cuenta el municipio La Sierpe en la Provincia de Sancti Spiritus. Para ello se utilizaron los siguientes métodos de investigación:

- Derecho Comparado: aseguró el cotejo de las normas jurídicas relacionadas con las zonas costeras y su manejo integrado en otros países, identificando las diferencias y semejanzas con la base jurídica de esta temática en Cuba y además aseguró el cotejo de las normas jurídicas derogadas y vigentes relacionadas con las zonas costeras y su manejo integrado en Cuba a partir de 1959.

- Inducción-deducción: aseguró arribar a conclusiones a partir de la información recopilada sobre las zonas costeras desde una dimensión jurídica.

- Exegético-Jurídico: proporcionó el análisis de los preceptos de las principales normas internacionales que regulan aspectos importantes de las Zonas Costeras.

- Revisión de literatura: se llevó a cabo en la mayor parte de la investigación, con el examen de varios textos de autores cubanos y extranjeros, que ya investigaron sobre este tema.

- Observación: permitió comprobar los daños existentes en el ecosistema y referidos en esta investigación.

\section{Resultados y discusión}

\section{Efectos provocados por la ausencia de un programa de manejo integrado en la zona costera del municipio La Sierpe}

El deterioro de la costa del municipio La Sierpe, ha estado influenciado por la ausencia de programas integrados para su manejo que favorecieran su evaluación, conservación y uso sostenible. Los efectos provocados por la ausencia del manejo integrado en la zona costera de La Sierpe, se resumen en tres grupos: deterioro de los recursos naturales; vulnerabilidades de la actividad económica y las prestaciones de servicio; pérdida de la diversidad biológica y efectos del cambio climático. 


\section{Deterioro de los recursos naturales en la zona costera de La Sierpe}

Dentro de los recursos naturales que ameritan la evaluación de los efectos causados por la ausencia del programa de manejo integrado de la zona costera se definen:

- Los bosques.

- Las aguas terrestres y marinas.

- Los suelos como uno de los componentes fundamentales de la tierra.

\section{Problemática de los bosques protectores}

El municipio de la Sierpe registra un índice de boscosidad de 9,9\%, cifras que por sí misma advierten la problemática existente en relación con la deforestación en el municipio. Al compararlo con el registro provincial que presenta un índice de boscosidad de $18,3 \%$, resulta significativa esta diferencia, pues se encuentra a solo el $50 \%$ de la media provincial y que a su vez está muy por debajo de los parámetros del país, con una superficie cubierta de bosques del $28,66 \%$, con un comportamiento de la media nacional del 25\%. Resulta en extremo preocupante que la zona costera sólo representa un 3.5\% del mencionado índice de boscosidad, ya que por el paso del tiempo y los efectos negativos asociados a fenómenos meteorológicos, los bosques de manglares existentes han ido deteriorándose.

Una reflexión en torno al tipo de zona costera de La Sierpe, según las clasificaciones definidas en el artículo 4 inciso d) del Decreto Ley 212/200o, atendiendo a su estructura y configuración, está considerada una costa baja de manglar. Las costas que reciben esta clasificación, exigen como su propio nombre lo indica, elevada presencia de mangle, que por sus características constituyen una vegetación imprescindible, para el cumplimiento de las funciones de protección contra el viento, inundaciones costeras por penetraciones del mar, intrusión salina y conservación de los ecosistemas costeros.

De igual manera la Ley No. 85/98 “Ley Forestal”, en el artículo 15 recoge la clasificación de los bosques según los elementos de orden físico, biológico, ecológico, social y económico. El inciso b) contempla los bosques de protección, como aquellos cuya superficie debe ser conservada permanentemente, para proteger los recursos renovables a los que estén asociados. Se añade en el artículo 18 del propio cuerpo legal, que los bosques protectores, de acuerdo con sus funciones esenciales se categorizan, distinguiendo dentro de ellos, los bosques protectores del litoral, que por su propia naturaleza y propiedades se conforman de diferentes especies de mangle.

Sin embargo, es evidente el deterioro del ecosistema manglar. La primera línea de costas se encuentra muy deforestada, al extremo que en la actualidad existe aproximadamente $1 \mathrm{~km}$ de costa, en que es nula la presencia del mangle. Se advierte la mortalidad o daño fisiológico del mangle, dado por el insuficiente aporte de agua dulce 
y nutriente. Esta problemática afecta la altura y densidad de los bosques de mangle, ya que lo que más recibe es agua muy salada, con un contenido muy bajo en nutrientes, provocando en los mangles el predominio de pequeña talla, achaparrados o enanos.

Por su parte, la segunda línea de costa, que según los requerimientos de la norma jurídica mencionada anteriormente debe estar conformada por arbustos que no rebasen los tres metros de altura, formando bosques semideciduos tropicales, en la zona costera estudiada, vemos que se encuentra en lo fundamental la Palma Cana. Esta plantación posee como características naturales, que es muy resistente a los fenómenos atmosféricos y tolerantes a los vientos salinos, la sequía y el encharcamiento de los suelos. Sin embargo no se ha logrado que las mismas conformen bosques protectores de la zona costera, ya que se encuentran dispersos con afectaciones en su población y limita su función de contención a los fenómenos meteorológicos.

En el 2014 se concibió un plan de 105 hectáreas a reforestar, con un 100\% de cumplimiento, pero de ellas no se dedicó ninguna cifra al litoral costero. Similar comportamiento se refleja en las cifras del 2015, que de un plan de 43 hectáreas, el municipio logra el 33.3 hectáreas y ninguna dedicada a la recuperación de áreas boscosas deterioradas, ni tampoco las que están totalmente deforestadas en la zona costera. La ausencia del programa de manejo integrado de la zona costera ha limitado las acciones para la recuperación de los bosques protectores, prevalecido los intereses de los tenentes de esos terrenos, en lo fundamental las Unidades Básicas de Producción Cooperativas pertenecientes a la Empresa Agroindustrial de Granos "Sur del Jíbaro".

Se añade la situación existente con la tala ilegal e indiscriminada de los bosques de manglares, lo que contraviene lo estipulado en el artículo 27 de la Ley Forestal en relación con las prohibiciones de talas de explotación, en los bosques protectores de las fajas forestales a partir de la línea de costa. Como parte de la evaluación de esta problemática es necesario apuntar que ha existido un éxodo masivo de la población de los lugares próximos a la zona costera. Reflejo de ello es que existen más de $17 \mathrm{~km}$ de costas totalmente deshabitados, que constituyen condiciones que favorecen estas ilegalidades.

Los bosques de manglares son desbastados para intereses particulares, como es el uso en el represamiento de las lagunas costeras. Estas violaciones están favorecidas por no contar el municipio, con ningún inspector del cuerpo de guarda bosques, para asegurar la aplicación de las contravenciones ante estas infracciones. Tales argumentos se corroboran con los análisis estadísticos de la Oficina Municipal de Control y Cobros de Multas del municipio La Sierpe, pues en el año 2013, se registraron solamente siete multas por violaciones relacionadas el ecosistema de manglar. Más preocupante resulta que solo uno obedece a la tala ilegal de los árboles. No ha existido una política consecuente con el control y aplicación de las normas jurídicas ante las violaciones que se producen en la zona costera. 


\section{Problemática de las aguas terrestres y marinas con efectos vinculantes en la zona costera}

Las aguas terrestres y marinas, constituyen recursos naturales de especial interés en la zona costera del municipio La Sierpe, ya que la zona costera recibe el aporte de agua dulce proveniente del río Zaza segunda cuenca de interés nacional, así como el río Jatibonico del Sur, cuenca de interés provincial y arroyos. Sin embargo, se ha visto afectado por alteraciones en el régimen hídrico, apreciado por obstrucción de canales y el represamiento de las aguas terrestres sobre todo para riego de arroz, que limitan la llegada a las lagunas del agua dulce, provocando consecuencias negativas al ecosistema manglar, azolvamiento y salinización sobre todo por el represamiento del rio Zaza que constituye la vía de abastecimiento de agua para el cultivo del arroz. En la observación efectuada como parte de los métodos de investigación se advirtió la presencia de ruinas antiguas de un puente en mal estado, que obstruye el paso del agua dulce. Se añade el azolvamiento producto de la alteración del aporte hídrico en la laguna.

La ausencia del programa de manejo integrado de la zona costera, ha propiciado el predominio de intereses económicos, sin evaluación de las afectaciones que se provocan al medio ambiente, que se refleja en la inadecuada política económica para sostener los renglones productivos. Las unidades productivas pertenecientes a la Empresa Agroindustrial de Granos "Sur del Jíbaro", poseen 20,130 hectáreas destinadas a la ganadería mayor y menor, sumado a propietarios de ganado vacuno, en áreas aledañas a las cuencas hidrográficas, ríos y arroyos. En la práctica y en aras de garantizar el agua necesaria para el sostenimiento del ganado, proceden a adoptar como medida alternativa para apalear los efectos de la sequía, el traslado del ganado para esos lugares, lo que constituye un foco contaminante de las aguas terrestres.

Se añaden los efectos provocados por la producción porcina que generan gran cantidad de aguas residuales con elevada carga contaminante. En las cercanías de la zona costera se ubica la Unidad Empresarial de Base Tamarindo, perteneciente a la Empresa Porcina Sancti Spíritus, que presenta dificultades en el sistema de tratamiento de las aguas residuales. También afecta la crianza de cerdos en traspatios de las viviendas en asentamientos muy próximos a la zona costera, que no cuentan con ningún sistema de vertimiento de los residuales líquidos y sólidos, que se incorporan directamente sobre todo como parte del fenómeno de escurrimiento de las aguas terrestres hacia el mar.

En cuanto a las aguas marinas la zona costera posee lagunas caracterizadas por sufrir fluctuaciones relativamente grandes en la salinidad y temperatura. El represamiento de ríos y otros cursos de agua ha conducido a la salinización y acumulación de sedimentos con la consecuente reducción del espejo de agua, con las consecuencias económicas de los recursos pesqueros en la zona. Existe penetración de la cuña salina, afectando ecosistemas productivos y alteración del régimen hídrico. En la observación 
efectuada durante la investigación, es casi inexistente la presencia de aves volando en el entorno costero, lo que advierte síntomas de contaminación de las aguas marinas.

Los diques han cortado o limitado el intercambio de agua produciendo daños a la ecología de algunas lagunas costeras y mortalidad o daño fisiológico del mangle. Los campos de arroz vierten sus aguas cargadas de nutrientes en la zona costera, provocando niveles importantes de eutrofización, con todas sus consecuencias ecológicas debido al elevado contenido de materia orgánica y nutriente. Los canales encargados de asegurar el vertimiento hacia el mar, carecen un canal transversal, que mitigue los efectos que provoca la entrada del agua residual altamente contaminada a la zona costera.

\section{Situación de los suelos del ecosistema productivo aledaño a la zona costera}

El análisis de la problemática de los suelos, posee un efecto vinculante con los objetivos económicos que caracterizan al municipio la Sierpe dedicado en lo fundamental a la producción de arroz. Para el cumplimiento de tales fines se creó La Empresa Agroindustrial de Granos "Sur del Jíbaro", que tiene una extensión de 83875 hectáreas, de las cuales se dedican al cultivo del arroz 27217 hectáreas y 2050 hectáreas a los cultivos varios. El computo de las áreas afectadas por sales en las diferentes Unidades Básicas de Producción Cooperativa, según la última evaluación efectuada por los especialistas de esa entidad, advierten su incremento progresivo desde el primer análisis efectuado en 1980 y su última evaluación en el 2011.

Las áreas afectadas se ubican en Mapo, Peralejo, Romero, Sur del Jíbaro, Cedro y las Nuevas. Dentro de los suelos afectados por la salinidad los índices más críticos se concentran en Mapos, Sur del Jibaro y Las Nuevas, que coinciden por ser terrenos aledaños a la zona costera y ante la deforestación de los bosques protectores, los efectos del deterioro medioambiental se reciben directamente al ecosistema productivo. Se añade a esta problemática el mal manejo de los suelos, la elevación del manto freático, la intrusión eólica y el mal drenaje.

Los análisis cuantitativos y cualitativos relacionados con los bosques protectores, las aguas terrestres, marinas y los suelos pertenecientes a las áreas cultivables del municipio La Sierpe, denotan la falta de integración de las entidades y organismos que cumplen funciones estatales para asegurar el uso y proyección de los recursos naturales con efecto vinculante en la zona costera. En consecuencia las limitaciones en la proyección territorial para el establecimiento e implementación de un programa de manejo integrado, con acciones dirigidas a la mitigación del deterioro medioambiental, han provocado efectos negativos en los recursos naturales de la zona costera de La Sierpe. 


\section{Pérdida de la diversidad biológica y efectos del cambio climático}

En el artículo 84 de la Ley del Medio Ambiente, se hace extensivo el mandato de conservar la diversidad biológica nacional y la utilización sostenible de sus componentes. En el artículo 85 se asegura la tutela jurídica de forma especial a las especies de carácter endémico que se encuentren, en peligro o vías de extinción, las representativas de los ecosistemas. En el caso de la zona costera de La Sierpe teniendo en cuenta las irregularidades que se han presentado en mal adopción de medidas preventivas y correctivas, para mitigar los efectos de la perdida de la diversidad biológica se reporta el retroceso en la presencia de especias endémicas del mar Caribe, como la cotorra y el perico.

La falta de transparencia, el cambio de coloración, los malos olores y la falta de la fauna asociada que huye de estas aguas, en las zonas eutróficas, producen una disminución del valor paisajístico de esta zona. La pérdida de diversidad de la fauna acuática disminuye el interés pesquero, comercial o deportivo de la zona. Los daños causados por la mortandad de los manglares que por su propia naturaleza constituyen refugio y reservorio de especies de la fauna terrestre marina, limita los procesos de reproducción de otros organismos que se desarrollan alrededor de las raíces como son peces típicos de la laguna costera como son róbalos, mojarras y pargos ,así como crustáceos.

Respecto a la pérdida de la diversidad biológica se evidencian los impactos negativos producto de la introducción de especies exóticas invasoras y su diseminación tales como: marabú (Leptoptilos crumeniferus), leucaena (Leucaena leucocephala), casuarina (Casuarina equisetifolia), en cuanto a la flora y la presencia de la claria (Pez gato), santanilla (Wasmannia auropunctata), en la fauna de aguas dulces. Se añade la presencia de búfalos silvestres (Bubalos Arnee) que causan efectos nocivos en las plantas protectoras de las cuencas hidrográficas y las aguas, ya que utilizan las áreas de las cuencas hidrográficas como medio para satisfacer sus necesidades de consumir agua ante las manifestaciones de intensa sequía.

Los efectos del cambio climático se observan en la gran erosión existente y el avance del mar hacia la tierra, lo que se define como aumento del nivel del mar. Las afectaciones en los manglares aceleran el proceso de erosión de la costa, provocada por el oleaje, el viento y las corrientes costeras. La deforestación impide la protección que los manglares brindan a la población e infraestructuras costeras contra las violentas penetraciones del mar y el efecto de los huracanes. Provoca mayor acción abrasiva del mar sobre las costas que han perdido la protección, con la consecuente disminución de las precipitaciones y los impactos negativos de los huracanes.

Otros de los problemas son los riesgos de inundaciones, provocado por los peligros de inundación por eventos lluviosos de la provincia el peligro alto aparece localizado en el municipio La Sierpe por la presencia de suelos impermeables, la escasa altitud 
sobre el nivel del mar y la planicidad del relieve. Se añade los planos de inundación del río Zaza y el vertimiento de aguas hacia poblados del mencionado municipio. Por su parte el río Jatibonico del Sur que incide en la Derivadora Sur del Jibaro.

Los asentamientos con mayor peligro ante inundaciones por lluvias intensas para un período de retorno de 50 años son: San Fernando, Pueblo Nuevo, Natividad, Mapos, Canta Rana, Aguachile, La Sierpe Vieja, la Sierpe, Guanalalto, La Chorrera, Brígidos, Boquerones I y II, Peralejo, Heriberto Orellanes, 26 de Julio, Las Nuevas, la Loma, El Jíbaro y Los Ferreiros.

La proporcionalidad del riesgo con la intensidad del fenómeno natural, advierte el nivel de vulnerabilidad existente en la zona costera del municipio La Sierpe. Condiciona la magnitud de las pérdidas al impactar un fenómeno meteorológico de gran intensidad, el cual puede ocasionar una situación de desastre en un área determinada. Las áreas de riesgo alto se incrementan en los Consejos Populares: Heriberto Orellanes, Mapos, Jíbaro y Las Nuevas.

Otro de los efectos es la penetración del mar, evento muy peligroso, que se produce al soplar el viento tormentoso producido por eventos ciclónicos, sures y frentes fríos sobre la superficie marina. El agua de mar que traslada la surgencia encuentra a su paso la desembocadura de un río, el agua marina acumulada llega a un punto crítico de ruptura con el agua dulce y asciende río arriba en forma de onda provocando fuertes inundaciones. Cuando este fenómeno se ha producido en la zona costera de La Sierpe ha provocado inundaciones, con graves pérdidas económicas, y peligrosidad para la vida humana, en los asentamientos rurales de Mapos, Natividad, Pueblo Nuevo, Agua Chile y San Fernando.

\section{Conclusiones}

Los efectos negativos provocados en los recursos naturales, la actividades económica, la prestación de los servicios, la perdida de la diversidad biológica y el cambio climático permiten comprender que ha existido inadecuado manejo de la zona costera como ecosistema frágil. Entre los factores que han influido en la problemática existente, se identifica la carencia un programa de manejo integrado de la zona costera. En consecuencia han predominado en el análisis de la problemática de la zona costera, los intereses de cada organismo o entidad en particular, y no en la consecución de acciones que produzcan mejor impacto en la zona costera. No se concretan acciones preventivas y correctivas ajustadas a las particularidades del territorio y es inadecuado el uso y la protección de la zona costera, todo lo que trae un consigo un efecto desfavorable y el consecuente deterioro medioambiental. 


\section{Lista de referencias}

Asamblea Nacional del Poder Ciudadano (1976). Constitución de la república de Cuba. La Habana, Cuba.

Asamblea Nacional del Poder Ciudadano (1981). Ley No.33 Protección del Medio Ambientey el Uso Racional de los Recursos Naturales. Publicado en la Gaceta Oficial Ordinaria No. 17 del 12 de febrero de 1981 . La Habana, Cuba.

Asamblea Nacional del Poder Ciudadano (1998). Ley No. 85/98 "Ley Forestal". Publicado en la Gaceta Oficial de la República de Cuba. Edición Ordinaria No. 46, del 31 de Agosto 31 de 1998. La Habana, Cuba.

Asamblea Nacional del Poder Ciudadano (1994). Decreto Ley No.147. De la reorganización de los organismos de la administración central del estado. Publicado en la Gaceta Oficial No. 2, del 21 de abril de 1994. La Habana, Cuba.

Asamblea Nacional del Poder Ciudadano (2000). Decreto Ley No.212. Gestión Integrada de la Zona Costera. Publicado en la Gaceta Oficial Ordinaria No. 68, del. 14 de julio del 2000). La Habana, Cuba.

Consejo de Estado (1980). Decreto Ley No.31 de 10 de enero de 1980, disponible en: http://www.gacetaoficial.cu/html/pdf/GO_O_022_2006.pdf

Pigretti, E. (1992). Ambiente, Energía y Derecho. Facultad de Derecho y Ciencias Sociales. Universidad de Buenos Aires, 3. 